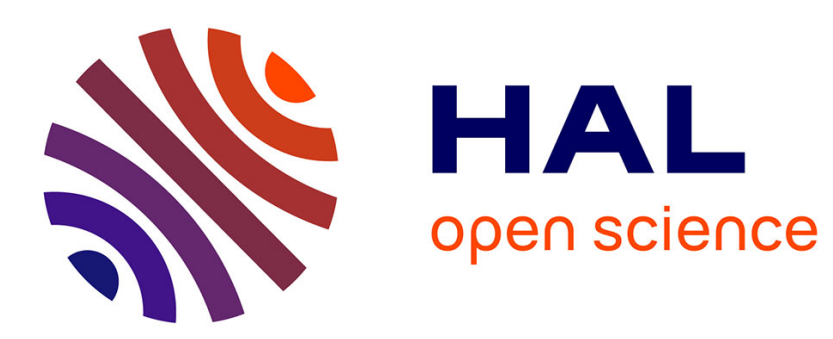

\title{
Digital Estimation and Compensation of I/Q Imbalance for Full-Duplex Dual-Band OFDM Radio
}

Zhaowu Zhan, Guillaume Villemaud, Florin Hutu, Jean-Marie Gorce

\section{To cite this version:}

Zhaowu Zhan, Guillaume Villemaud, Florin Hutu, Jean-Marie Gorce. Digital Estimation and Compensation of I/Q Imbalance for Full-Duplex Dual-Band OFDM Radio. PIMRC 2014, Sep 2014, Washington, United States. hal-01090698

\section{HAL Id: hal-01090698 \\ https://inria.hal.science/hal-01090698}

Submitted on 4 Dec 2014

HAL is a multi-disciplinary open access archive for the deposit and dissemination of scientific research documents, whether they are published or not. The documents may come from teaching and research institutions in France or abroad, or from public or private research centers.
L'archive ouverte pluridisciplinaire HAL, est destinée au dépôt et à la diffusion de documents scientifiques de niveau recherche, publiés ou non, émanant des établissements d'enseignement et de recherche français ou étrangers, des laboratoires publics ou privés. 


\title{
Digital Estimation and Compensation of I/Q Imbalance for Full-Duplex Dual-Band OFDM Radio
}

\author{
Zhaowu Zhan, Guillaume Villemaud, Florin Hutu and Jean-Marie Gorce \\ Université de Lyon, INRIA \\ INSA-Lyon, CITI-INRIA, F-69621, Villeurbanne, France \\ Email: zhaowu.zhan@insa-lyon.fr
}

\begin{abstract}
With the increasing demand of wireless applications, current radio transceivers are challenged by the requirement of high data rate and high flexibility. Full-Duplex Dual-Band OFDM radio transceiver is a very promising radio technique to approach this goal. However, the mutual undesirable signal leakages due to the I/Q imbalance in the Full-Duplex Dual-Band RF front-end lead to a significant performance degradation in the radio link. In this paper, a practical and suitable digital I/Q imbalance estimation and compensation method for mitigating the $I / Q$ imbalance in this flexible radio transceivers is developed and evaluated. The developed $I / Q$ imbalance estimation method is based on the character of the frequency-flat-fading of the selfinterference channel. The ADS-Matlab co-simulation results show that the developed digital compensation method can significantly reduce the impact of the I/Q imbalance on the Full-Duplex DualBand OFDM radio receivers.
\end{abstract}

\section{INTRODUCTION}

The growing demands for various multimedias and increasingly pervasive wireless radios call for the development of advanced and flexible radio transceivers to support high data rate and different wireless standards over limited radio resources in the future wireless network. However, seeking a flexible and radio agile transceiver is always a big challenge. Fortunately, the noticeable gain of radio link capacity and the capability of processing two different types of signals of the Full-Duplex Dual-Band radio [1] promote it as a flexible radio front-end. Furthermore, OFDM-based capabilities of sensing and spectrum shaping together with its flexibility make it probably the best transmission technology for the flexible radios [2]. Therefore, the Full-Duplex Dual-Band OFDM radio transceivers based on the RF front-end proposed in [1] would be the flexible radio transceiver we are exploring. However, this radio transceiver suffers from RF impairments such as I/Q imbalance which causes undesired mutual signal leakage. In order to implement a high performance Full-Duplex DualBand OFDM radio transceiver, it is necessary to estimate and compensate the I/Q imbalance beforehand.

Down-converting the radio frequency (RF) signal to baseband signal is a fundamental function of all receiver RF frontend. Up to date, there are mainly two RF architectures to convert the RF signal to baseband, i.e. through intermediate frequency (IF) or via direct-conversion (Zero-IF). Based on this, the radio receivers are classified into Zero-IF receiver and superheterodyne (with IF) receiver. For different types of radio receivers, there are different literatures for addressing the I/Q imbalance. Due to lots of literatures on that topic, we will just put out some significant examples.

For Zero-IF receivers, the authors [3] proposed I/Q imbalance estimation based on the design of special pilot tones and least mean squares (LMS) algorithm. This technique requires the modification of the training signal and suffers from the huge number of OFDM training symbols and low convergence rate. [4] proposed special training sequence design for the I/Q imbalance estimation. Another I/Q imbalance estimation technique based on the redesign of the preamble proposed by [5] requires the modification of the preamble of IEEE 802.11a. [6] and [7] proposed I/Q imbalance compensation based on the assumption that the adjacent subcarriers are highly correlated. Windisch et al. [8] proposed the I/Q imbalance estimation based on the statistical analysis of data symbols, i.e. blind estimation. This method requires large number of data symbols to obtain a good I/Q imbalance estimation.

For the superheterodyne receivers, [9] proposed to employ two different long training symbols instead of two identical long training symbols to carry out the channel and I/Q imbalance estimation. However, the two long training symbols are identical in the practical implementation of IEEE $802.11 \mathrm{~g}$. Then, the authors further proposed an iterative algorithm by using both the training and data OFDM symbols. Blind I/Q imbalance estimation based on the two received baseband signals (uncompensated desired and image signals) were presented in [10] and [11]. This approach relies on the assumption that the desired and image signals are zero-mean wide-sense stationary random processes and mutually uncorrelated, which put high requirement on the signal design.

In this paper, the radio receiver architecture we studied is Full-Duplex Dual-Band superheterodyne receiver. The available I/Q imbalance estimation and compensation methods are not suitable to address the I/Q imbalance faced in this flexible radio receiver. Therefore, we developed an advanced and practical digital I/Q imbalance estimation and compensation method for mitigating the I/Q imbalance existing in the FullDuplex Dual-Band RF front-end.

The rest of the paper is organized as follows: Section II presents the system model of Full-Duplex Dual-Band OFDM radio transceiver. Then, the algorithm of I/Q imbalance estimation and compensation is given in Section III. After that, the simulation results are presented in Section IV. Finally, we draw the conclusion in Section V. 


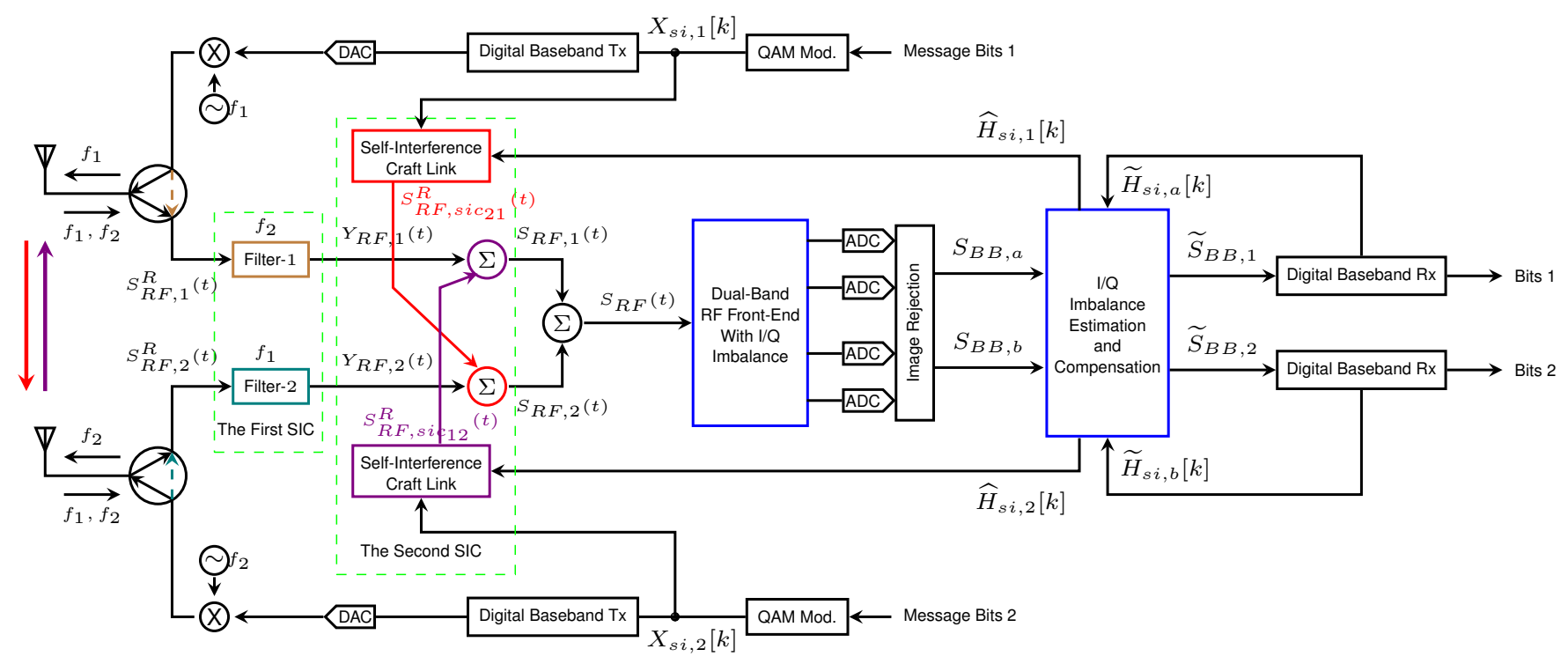

Fig. 1. Full-Duplex Dual-Band OFDM radio transceiver with I/Q imbalance compensation

\section{Full-Duplex DuAl-BAND OFDM RADio RECEIVER}

\section{A. General Signal Model}

In this section, we present the radio architecture proposed in [1] with I/Q imbalance in the Full-Duplex Dual-Band RF front-end as shown in Fig. 1. The RF signals arrived at the $i^{\text {th }}$ antenna of the radio node plus the self-interference (SI) signal leaked from the imperfect circulator are expressed as

$$
\begin{aligned}
S_{R F, i}^{R}(t)= & S_{R F, i j}^{R}(t)+S_{R F, i i}^{R}(t)+S_{R F, s i_{i j}}^{R}(t) \\
& +a S_{R F, s i_{i i}}^{R}(t),\{i, j\} \in A
\end{aligned}
$$

where $S_{R F, i j}^{R}(t)$ and $S_{R F, i i}^{R}(t)$ are the received RF signal from the distant radio node with carrier frequency $f_{j}$ and $f_{i}$ respectively, $S_{R F, s i_{i j}}^{R}(t)$ denotes the inter RF SI signal from the other antenna of the local radio node, $S_{R F, s i_{i i}}^{R}(t)$ represents the inner RF SI signal transmitted and received by the same antenna and circulator, $a$ being the inner-SI factor including multi-path reflection factor and inner leakage factor of the circulator and $A \triangleq\{i \in[1,2], j \in[1,2], i+j=3\}$.

Following the circulator, simple BPFs (Band Pass Filter) are employed to construct the first self-interference cancellation (SIC) which filter out the inner SI. At the same time, the RF signal having the same carrier frequency as this inner SI signal will also be filtered out. Then, the signals after the first SIC can be represented by

$$
Y_{R F, i}(t)=S_{R F, i j}^{R}(t)+S_{R F, s i_{i j}}^{R}(t),\{i, j\} \in A
$$

As we can see, the desired signal $S_{R F, i j}^{R}$ from the distant radio node is superposed by the inter SI signal $S_{R F, s i_{i j}}^{R}$. Due to the short distance between the antennas of the local radio node, the SI signal is hundreds of thousands of times of the signal of interest. In order to avoid the saturation of ADC, the strong SI should be suppressed to a tolerable level at first.
Therefore, we craft the RF cancellation signal and implement the cancellation as in [12]. The remaining RF signal after the two stages of SIC will be

$$
\begin{aligned}
S_{R F, i}(t) & =Y_{R F, i}^{R}(t)-S_{R F, s i c_{i j}}^{R}(t),\{i, j\} \in A \\
& =S_{R F, i j}^{R}(t)+\underbrace{S_{R F, s i_{i j}}^{R}(t)-S_{R F, s i c_{i j}}^{R}(t)}_{\text {residual self-interference }}
\end{aligned}
$$

That means the signal of interest is corrupted by the residual SI caused by the imperfection of the second SIC. The blended signal consisting of two corrupted signals to go through the Dual-Band RF front-end is

$$
S_{R F}(t)=S_{R F, 1}(t)+S_{R F, 2}(t)
$$

where $S_{R F, 1}(t)$ and $S_{R F, 2}(t)$ are the RF signals with the carrier frequency $f_{2}$ and $f_{1}$ respectively.

\section{B. I/Q Imbalance Model}

In the Full-Duplex Dual-Band radio system, the double I/Q structure is utilized to down-convert the RF signal $S_{R F}(t)$. However, perfect I/Q matching is not available in practice. All of the analog components (such as mixer, filters and analog to digital converters) at radio receivers lead to the deviations from the desired $90^{\circ}$ phase shift and the desired equal gain in the I and the $\mathrm{Q}$ branch as shown in Fig. 2. Here, we use $\Delta A$ and $\Delta \phi$ to denote the amplitude imbalance and the phase imbalance respectively. Therefore, after down-converting the RF signals (4) via using Dual-Band RF front-end with I/Q imbalance [13], the two output baseband signals can be expressed as

$$
\begin{aligned}
& S_{B B, a}=\alpha S_{B B, 1}+\beta S_{B B, 2}^{*} \\
& S_{B B, b}=\alpha S_{B B, 2}+\beta S_{B B, 1}^{*}
\end{aligned}
$$

where $S_{B B, 1}$ and $S_{B B, 2}$ denote the equivalent baseband signals of $S_{R F, 1}(t)$ and $S_{R F, 2}(t)$ respectively, $(\cdot)^{*}$ represents 


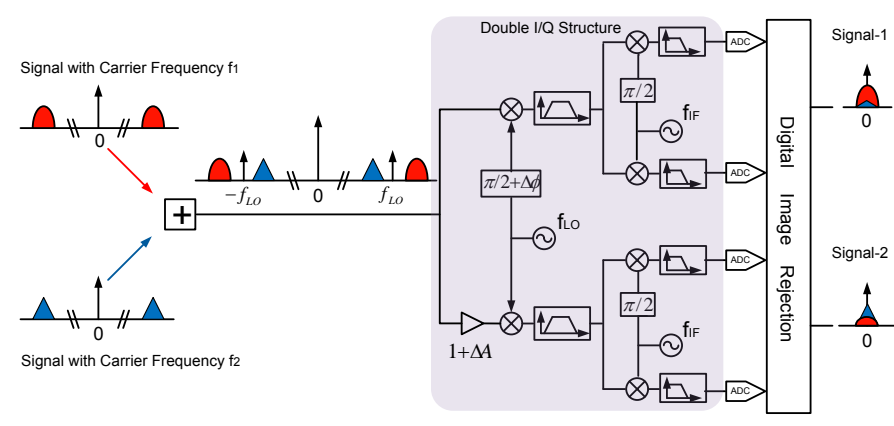

Fig. 2. Architecture of Dual-Band RF Front-End with I/Q imbalance

complex conjugation, $\alpha$ and $\beta$ are the I/Q imbalance coefficients which can be expressed as

$$
\begin{aligned}
& \alpha=\frac{1+(1+\Delta A) e^{-j \Delta \phi}}{2} \\
& \beta=\frac{1-(1+\Delta A) e^{+j \Delta \phi}}{2}
\end{aligned}
$$

where we can obtain $\alpha+\beta^{*}=\alpha^{*}+\beta=1$.

In our study, different radio nodes uses different time slots for transmitting the long training symbols to avoid the impact of the signals from the distant radio node on the SI channel estimation of the local radio node. Therefore, the baseband signals (5) (6) during the SI channel estimation could be represented by

$$
\begin{aligned}
& S_{B B, L T S, a}=\alpha S_{B B, L T S, 1}+\beta S_{B B, L T S, 2}^{*} \\
& S_{B B, L T S, b}=\alpha S_{B B, L T S, 2}+\beta S_{B B, L T S, 1}^{*}
\end{aligned}
$$

where $L T S$ denotes the Long Training Signal.

Then, the SI channel estimation is implemented via dividing the demodulated long training symbols without the null subcarriers by the $N_{n z}$ known training symbols. The SI channel coefficients $\widetilde{H}_{s i, a}[k]$ and $\widetilde{H}_{s i, b}[k]$ can be estimated according to (9) (10) by using least square (LS) algorithm as

$$
\begin{aligned}
\widetilde{H}_{s i, a}[k] & =\alpha \widetilde{H}_{s i, 1}[k]+\beta T^{\prime}[k] \cdot \widetilde{H}_{s i, 2}^{*}\left[N_{n z}-k+1\right] \\
\widetilde{H}_{s i, b}[k] & =\alpha \widetilde{H}_{s i, 2}[k]+\beta T^{\prime}[k] \cdot \widetilde{H}_{s i, 1}^{*}\left[N_{n z}-k+1\right]
\end{aligned}
$$

where $\widetilde{H}_{s i, 1}[k]$ and $\widetilde{H}_{s i, 2}[k]$ represent the estimated coefficient of the $k^{t h}$ subcarrier channel of the SI channel-1 and channel2 respectively, $\widetilde{H}_{s i, 1}\left[N_{n z}-k+1\right]$ and $\widetilde{H}_{s i, 2}\left[N_{n z}-k+1\right]$ are the mirror image of the channel $\widetilde{H}_{s i, 1}[k]$ and $\widetilde{H}_{s i, 2}[k]$, $T^{\prime}[k]=T[k] T\left[N_{n z}-k+1\right]$, ". " denotes component-wise vector multiplication.

From (5) (6) (11) (12), we can see that the I/Q imbalance not only makes the image signal superpose on each other but also corrupts the channel estimation including SI channel estimation and the channel estimation between different radio nodes. Therefore, the negative effect of the I/Q imbalance on the Full-Duplex Dual-Band OFDM radio includes: (i) reducing the SINR (signal to interference and noise power ratio) by causing extra interference noise superposed on the desired signal; (ii) significantly degrades the performance of SIC by causing the SI channel estimation error; (iii) the estimation error of the channel between different radio nodes will badly influence the OFDM equalization at the receiver. Therefore, it is highly necessary to mitigate the I/Q imbalance for the Full-Duplex Dual-Band OFDM radio transceiver.

\section{I/Q IMBALANCE ESTIMATION AND COMPENSATION}

In fact, if we know exactly the value of the I/Q imbalance parameters $\alpha$ and $\beta$, the two different types of signals could be separated completely based on

$$
\begin{aligned}
S_{B B, 1} & =\frac{\alpha^{*} S_{B B, a}-\beta S_{B B, b}^{*}}{|\alpha|^{2}-|\beta|^{2}} \\
S_{B B, 2} & =\frac{\alpha^{*} S_{B B, b}-\beta S_{B B, a}^{*}}{|\alpha|^{2}-|\beta|^{2}}
\end{aligned}
$$

However, $\alpha$ and $\beta$ are functions of $\Delta A$ and $\Delta \phi$ whose values are dependent on the performance of analog device in the RF front-end. Therefore, it is not easy to obtain the value of $\alpha$ and $\beta$, but we can estimate it by using the training sequences, which rely on the fact that $\Delta A$ and $\Delta \phi$ are quite stable.

\section{A. I/Q Imbalance Estimation}

In order to derive the optimal estimation of $\alpha$ and $\beta$, we first assume that the estimated I/Q imbalance parameters are $\widetilde{\alpha}$ and $\widetilde{\beta}$. With the assumed estimation values $\widetilde{\alpha}$ and $\widetilde{\beta}$, we can obtain the virtual estimated SI channels according to (11) (12) as

$$
\begin{aligned}
\widehat{H}_{s i, 1}[k] & =\frac{\widetilde{\alpha}^{*} \widetilde{H}_{s i, a}[k]-\widetilde{\beta} T^{\prime}[k] \cdot \widetilde{H}_{s i, b}^{*}\left[N_{n z}-k+1\right]}{|\widetilde{\alpha}|^{2}-|\widetilde{\beta}|^{2}} \\
\widehat{H}_{s i, 2}[k] & =\frac{\widetilde{\alpha}^{*} \widetilde{H}_{s i, b}[k]-\widetilde{\beta} T^{\prime}[k] \cdot \widetilde{H_{s i, a}^{*}}\left[N_{n z}-k+1\right]}{|\widetilde{\alpha}|^{2}-|\widetilde{\beta}|^{2}}
\end{aligned}
$$

Due to the short distance between the two antennas of the local radio node, the power of the line-of-sight path dominate the SI channel. Therefore, the SI channel is much more close to a frequency flat fading channel [14]. However, the SI channel is more like a frequency selective fading channel when the I/Q imbalance exists in the RF front-end [6] [7]. Therefore, the optimal estimated value of the I/Q imbalance should be $\left(\widetilde{\alpha}_{o}, \widetilde{\beta}_{o}\right)$ which can make the virtual estimated SI channel as flat as possible as the SI channel is. Based on this, we formulate the optimization problem as

$$
\left(\widetilde{\alpha}_{o}, \widetilde{\beta}_{o}\right)=\arg \min _{\widetilde{\alpha} \in C, \widetilde{\beta} \in C}\left\{\sum_{k}\left\|\widehat{H}_{s i, i}[k+1]-\widehat{H}_{s i, i}[k]\right\|^{2}\right\}
$$

Here, we define the criteria to measure how flat the frequency domain SI channel with I/Q imbalance is as

$$
M S E_{i}=\sum_{k}\left\|\widehat{H}_{s i, i}[k+1]-\widehat{H}_{s i, i}[k]\right\|^{2}, i \in\{1,2\}
$$

For this symmetric problem, we just study the case $M S E_{1}$. After replacing the $\hat{H}_{s i, i}[k]$ in (18) by (15), we can obtain the $M S E_{1}$ as

$$
M S E_{1}=\frac{\sum_{k}\left\|\widetilde{\alpha}^{*} \Delta \widetilde{H}_{s i, a}[k+1]-\widetilde{\beta} \Delta \widetilde{H}_{s i, b}^{\prime}[k]\right\|^{2}}{\left(|\widetilde{\alpha}|^{2}-|\widetilde{\beta}|^{2}\right)^{2}}
$$


where

$$
\begin{aligned}
\Delta \widetilde{H}_{s i, a}[k]= & \widetilde{H}_{s i, a}[k+1]-\widetilde{H}_{s i, a}[k] \\
\Delta \widetilde{H}_{s i, b}^{\prime}[k]= & T^{\prime}[k+1] \cdot \widetilde{H}_{s i, b}^{*}\left[N_{n z}-k\right] \\
& -T^{\prime}[k] \cdot \widetilde{H}_{s i, b}^{*}\left[N_{n z}-k+1\right]
\end{aligned}
$$

According to (11) (12), we can figure out that $\widetilde{\beta}$ is the key factor that makes the frequency flat fading SI channel be a frequency selective fading channel. And from (19), we can see that $M S E_{1}$ is function of $\widetilde{\beta}$. In order to obtain the optimal value of $\widetilde{\beta}$, we take the partial derivative of $M S E_{1}$ with respect to $\widetilde{\beta}$ as

$$
\frac{\partial M S E_{1}}{\partial \widetilde{\beta}}=\sum_{k} \frac{\partial\left\{\frac{\left\|\widetilde{\alpha}^{*} \cdot \Delta \widetilde{H}_{s i, a}[k]-\widetilde{\beta} \cdot \Delta \widetilde{H}_{s i, b}^{\prime}[k]\right\|^{2}}{\left(|\widetilde{\alpha}|^{2}-|\widetilde{\beta}|^{2}\right)^{2}}\right\}}{\partial \widetilde{\beta}}
$$

Because of $\left(|\widetilde{\alpha}|^{2}-|\widetilde{\beta}|^{2}\right)^{2} \approx 1$, even valid for high gain imbalance $(\Delta A=0.2)$ and phase imbalance $\left(\Delta \phi=10^{\circ}\right)$, (22) can be simplified as

$$
\frac{\partial M S E_{1}}{\partial \widetilde{\beta}} \approx \sum_{k} \frac{\partial\left\|\widetilde{\alpha}^{*} \cdot \Delta \widetilde{H}_{s i, a}[k]-\widetilde{\beta} \cdot \Delta \widetilde{H}_{s i, b}^{\prime}[k]\right\|^{2}}{\partial \widetilde{\beta}}
$$

We define $z_{k}=\left\|\widetilde{\alpha}^{*} \cdot \Delta \widetilde{H}_{s i, a}[k]-\widetilde{\beta} \cdot \Delta \widetilde{H}_{s i, b}^{\prime}[k]\right\|^{2}$ here. Because of $\widetilde{\beta}=\Re(\widetilde{\beta})+\jmath \Im(\widetilde{\beta}), z_{k}$ is actually function of $\Re(\widetilde{\beta})$ and $\Im(\widetilde{\beta})$. According to the Cauchy-Riemann equations

$$
\begin{gathered}
\sum_{k} \frac{\partial \Re\left(z_{k}\right)}{\partial \Re(\widetilde{\beta})}=\sum_{k} \frac{\partial \Im\left(z_{k}\right)}{\partial \Im(\widetilde{\beta})} \\
\sum_{k}-\frac{\partial \Re\left(z_{k}\right)}{\partial \Im(\widetilde{\beta})}=\sum_{k} \frac{\partial \Im\left(z_{k}\right)}{\partial \Re(\widetilde{\beta})}
\end{gathered}
$$

From the definition of $z_{k}$, we can know that $z_{k}$ is real value. That means $\Im(\widetilde{\beta})=0$. Then, (24) (25) can be rewritten as

$$
\begin{aligned}
\sum_{k} \frac{\partial \Re\left(z_{k}\right)}{\partial \Re(\widetilde{\beta})} & =0 \\
\sum_{k}-\frac{\partial \Re\left(z_{k}\right)}{\partial \Im(\widetilde{\beta})} & =0
\end{aligned}
$$

Based on (26) (27), the real and imaginary part of the optimal estimated $\widetilde{\beta}$ can be obtained as

$$
\begin{aligned}
& \Re\left(\widetilde{\beta}_{o}\right)=\frac{\sum_{k} \Re\left(\widetilde{\alpha}_{o} \cdot \Delta \widetilde{H}_{s i, a}^{*}[k] \cdot \Delta \widetilde{H}_{s i, b}^{\prime}[k]\right)}{\sum_{k}\left|\Delta \widetilde{H}_{s i, b}^{\prime}[k]\right|^{2}} \\
& \Im\left(\widetilde{\beta}_{o}\right)=\frac{-\sum_{k} \Im\left(\widetilde{\alpha}_{o} \cdot \Delta \widetilde{H}_{s i, a}^{*}[k] \cdot \Delta \widetilde{H}_{s i, b}^{\prime}[k]\right)}{\sum_{k}\left|\Delta \widetilde{H}_{s i, b}^{\prime}[k]\right|^{2}}
\end{aligned}
$$

Then, the optimal value $\widetilde{\beta}_{o}$ can be represented by

$$
\begin{aligned}
\widetilde{\beta}_{o} & =\Re\left(\widetilde{\beta}_{o}\right)+\jmath \Im\left(\widetilde{\beta}_{o}\right) \\
& =\frac{\sum_{k} \widetilde{\alpha}_{o}^{*} \cdot \Delta \widetilde{H}_{s i, a}[k] \cdot \Delta \widetilde{H}_{s i, b}^{\prime *}[k]}{\sum_{k}\left|\Delta \widetilde{H}_{s i, b}^{\prime}[k]\right|^{2}} \\
& =\widetilde{\alpha}_{o}^{*} \widetilde{\beta}_{1}
\end{aligned}
$$

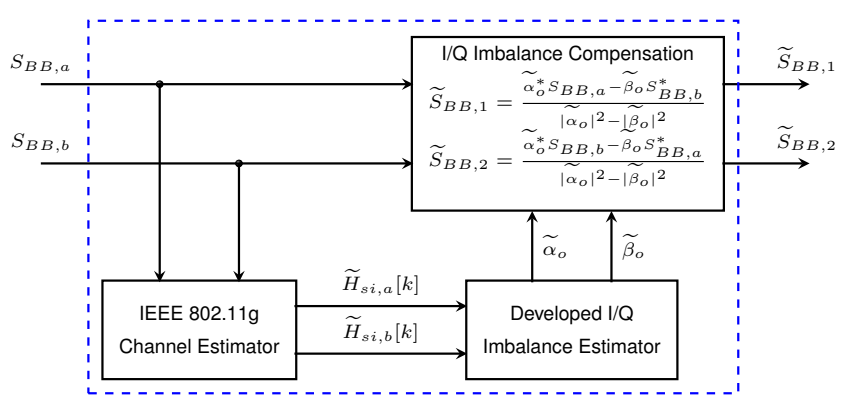

Fig. 3. Illustration of $\mathrm{I} / \mathrm{Q}$ imbalance estimation and compensation.

where $\widetilde{\beta}_{1}=\frac{\sum_{k} \Delta \widetilde{H}_{s i, a}[k] \cdot \Delta \widetilde{H}_{s i, b}^{\prime *}[k]}{\sum_{k}\left|\Delta \widetilde{H}_{s i, b}^{\prime}[k]\right|^{2}}$. That means $\widetilde{\beta}_{1}$ can be easily obtained by calculating the estimated coefficients sequences $\widetilde{H}_{s i, a}[k]$ and $\widetilde{H}_{s i, b}[k], k \in\left[1, N_{n z}\right]$ which are corresponding to the two SI channel estimations. Furthermore, $\widetilde{\alpha}_{o}+\widetilde{\beta}_{o}^{*}=1$, so the optimal estimated values of the I/Q imbalance parameters can be obtained as

$$
\begin{aligned}
& \widetilde{\alpha}_{o}=\frac{1}{1+\widetilde{\beta}_{1}} \\
& \widetilde{\beta}_{o}=1-\widetilde{\alpha}_{o}^{*}
\end{aligned}
$$

\section{B. I/Q Imbalance Compensation}

With the estimated I/Q imbalance parameters available, the I/Q imbalance in the Full-Duplex Dual-Band RF front-end can be compensated as in Fig. 3. The SI channel $\widehat{H}_{s i, 1}[k]$ and $\widehat{H}_{s i, 2}[k]$ can also be obtained by solving (15) (16). Then, the two baseband signals can be separated by solving (5) (6) as

$$
\begin{aligned}
\widetilde{S}_{B B, 1} & =\frac{\widetilde{\alpha}_{o}^{*} S_{B B, a}-\widetilde{\beta}_{o} S_{B B, b}^{*}}{\left|\widetilde{\alpha}_{o}\right|^{2}-\left|\widetilde{\beta}_{o}\right|^{2}} \\
\widetilde{S}_{B B, 2} & =\frac{\widetilde{\alpha}_{o}^{*} S_{B B, b}-\widetilde{\beta}_{o} S_{B B, a}^{*}}{\left|\widetilde{\alpha}_{o}\right|^{2}-\left|\widetilde{\beta}_{o}\right|^{2}}
\end{aligned}
$$

where $S_{B B, a}$ and $S_{B B, b}$ are the received baseband signals corrupted by the I/Q imbalance, $\widetilde{S}_{B B, 1}$ and $\widetilde{S}_{B B, 2}$ are the baseband signals corresponding to the pure baseband signals $S_{B B, 1}$ and $S_{B B, 2}$ respectively after the I/Q imbalance compensation.

\section{Simulation Results ANd Discussion}

In order to construct a system level simulation platform for Full-Duplex Dual-Band OFDM radio transceiver, we model the RF front-end and baseband system on the ADS (Advanced Design System, Agilent Technologies) and part of digital signal processing and algorithm implementation on Matlab. The wireless channel between different radio nodes is typical WLAN channel. The system parameters are chosen as in Table I according to the IEEE $802.11 \mathrm{~g}$ WLAN standard.

\section{A. Simulation Results}

Before presenting the BER (Bit Error Rate) performance of the radio system, we detail the notations in the Fig. 4. "FDDB WiFi-1" and "FDDB WiFi-2" denote the Full-Duplex Dual-Band IEEE 802.11g signal with carrier frequency $f_{1}$ 
TABLE I

SYSTEM PARAMETERS

\begin{tabular}{cc}
\hline \hline Parameter & Value \\
\hline Bandwidth & $20 \mathrm{MHz}$ \\
Carrier frequency $f_{1}$ & $2.4 \mathrm{GHz}$ \\
Carrier frequency $f_{2}$ & $2.0 \mathrm{GHz}$ \\
Total number of subcarriers & 52 \\
Number of data subcarriers & 48 \\
Number of pilot subcarriers & 4 \\
Bit rate & $36 \mathrm{Mbps}$ \\
IFFT/FFT period & $3.2 \mathrm{us}$ \\
GI duration & $0.8 \mathrm{us}$
\end{tabular}

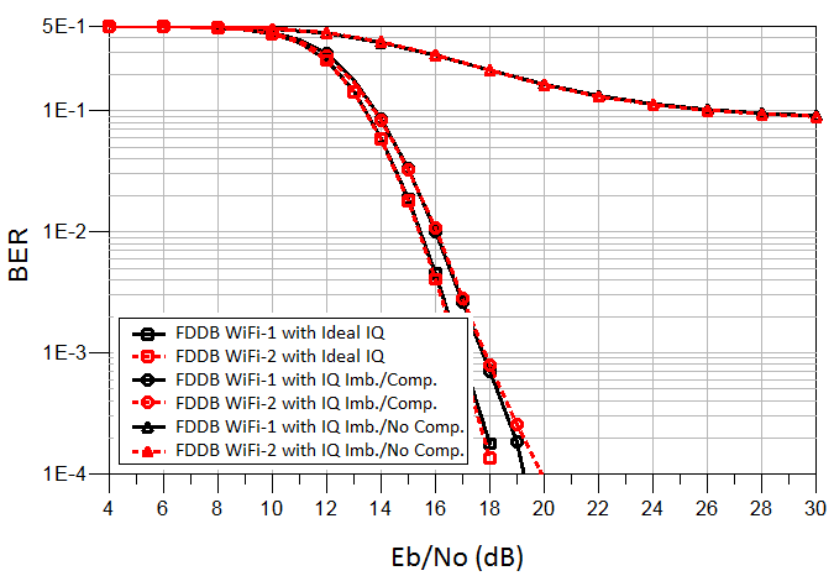

Fig. 4. The BER of Full-Duplex Dual-Band IEEE 802.11g wireless system, with $\mathrm{I} / \mathrm{Q}$ imbalance $\left(\Delta A=0.2, \Delta \phi=10^{\circ}\right)$.

and $f_{2}$ respectively; "with Ideal IQ" denote the radio receiver without I/Q imbalance; "with IQ Imb./Comp." represents the radio receiver with I/Q imbalance and also with I/Q imbalance compensation; "with IQ Imb./No Comp." represents the radio receiver with I/Q imbalance but without I/Q imbalance compensation. Then, we can see that, there is a BER $=10^{-1}$ floor when I/Q imbalance $\left(\Delta A=0.2, \Delta \phi=10^{\circ}\right)$ exists in the Full-Duplex Dual-Band RF front-end. That means the studied radio receiver quality is almost destroyed by the I/Q imbalance. However, this RF impaired radio system can be repaired by the I/Q imbalance compensation method proposed in this paper. As we can see, there is around $1 \sim 1.5 \mathrm{~dB}$ gap between impaired RF front-end with I/Q compensation and the ideal I/Q RF front-end system when $B E R=10^{-4}$. Therefore, the impact of I/Q imbalance on the Full-Duplex Dual-Band OFDM radio receiver has been significantly reduced by utilizing the I/Q imbalance compensation algorithm.

\section{B. Discussion}

In the simulation setup, we make the transmit power of each signal equal. Therefore, the two different types of signals from the distant radio node will arrive at the antennas of the local radio node with almost equal strength, which relies on the fact that the radio node can be seen as a point compared to the distance between the two radio nodes. In this scenario, each signal is superposed by the same power of the other image signal. Therefore, the two signal with different carrier frequency are received with the same performance. However, it is hard for this radio receiver to perform the same with another scenario: the two received RF signal with different power level. Additionally, the first SIC using BP-filter indeed relaxes the high requirements of isolation on the circulator.

\section{CONCLUSION}

Full-Duplex Dual-Band OFDM radio is a flexible radio, which could provide users flexible radio connection and high aggregate throughput. However, the I/Q imbalance in the FullDuplex Dual-Band RF front-end causes signal leakage to each other. These mutual leaked signals significantly degrade the reception performance of the Full-Duplex Dual-Band OFDM radio system. In order to realize the viable performance of this radio system, we developed an I/Q imbalance compensation algorithm based on the character of frequency-flat-fading of the SI channel. Excellent performance of this algorithm render it a suitable I/Q imbalance estimation and compensation method for the Full-Duplex Dual-Band OFDM radio receivers.

\section{REFERENCES}

[1] Z. Zhan, G. Villemaud, F. Hutu, and J.-M. Gorce, "Full-Duplex DualBand radio dedicated to flexible radio communications," INRIA Research Report, 2014.

[2] H. Mahmoud, T. Yucek, and H. Arslan, "OFDM for cognitive radio: merits and challenges," IEEE Wireless Communications, vol. 16, no. 2, pp. 6-15, 2009.

[3] H. Tarighat, R. Bagheri, and A. Sayed, "Compensation schemes and performance analysis of iq imbalance in OFDM receivers," IEEE Transactions on Signal Processing, vol. 53, no. 8, pp. 3257-3268, 2005.

[4] I. Sohn, E. Jeong, and Y. Lee, "Data-aided approach to I/Q mismatch and DC offset compensation in communication receivers," IEEE Communications Letters, vol. 6, no. 12, pp. 547-549, 2002.

[5] M. Windisch and G. Fettweis, "Preamble design for an efficient I/Q imbalance compensation in OFDM direct-conversion receivers," in Proc. 10th International OFDM Workshop, 2005.

[6] J. Tubbax, B. Come, L. der Perre, S. Donnay, M. Engels, H. Man, and M. Moonen, "Compensation of IQ imbalance and phase noise in OFDM systems," IEEE Transactions on Wireless Communciations, vol. 4, no. 3, pp. 872-877, 2005.

[7] J. Yu, M. Sun, T. Hus, and C. Lee, "A novel technique for I/Q imbalance and CFO compensation in OFDM systems," in Proc. IEEE International Symposium on Circuit and Systems, 2005.

[8] M. Windisch and G. Fettweis, "Standard-independent I/Q imbalance compensation in OFDM direct-conversion receivers," in Proc. 9th International OFDM Workshop, 2004.

[9] S. Traverso, M. Ariaudo, I. Fijalkow, J. Gautier, and C. Lereau, "Decision-directed channel estimation and high I/Q imbalance compensation in OFDM receivers," IEEE Transactions on Communciations, vol. 57, no. 5, pp. 1246-1249, 2009.

[10] G. Gil, Y. Kim, and Y. Lee, "Non-data-aided approach to I/Q mismatch compensation in low-IF receivers," IEEE Transactions on Signal Processing, vol. 55, no. 7, pp. 3360-3365, 2007.

[11] M. Windisch and G. Fettweis, "Blind I/Q imbalance parameter estimation and compensation in low-IF receivers," in Proc. First International Symposium on Control, Communications and Signal Processing, 2004.

[12] Z. Zhan, G. Villemaud, and J.-M. Gorce, "Analysis and reduction of the impact of thermal noise on the Full-Duplex OFDM radio," in Proc. IEEE Radio and Wireless Symposium, 2014.

[13] I. Burciu, G. Villemaud, and J. Verdier, "Multiband simultaneous reception front-end with adaptive mismatches correction algorithm," in Proc. 20th IEEE International Symposium on Personal, Indoor and Mobile Radio Communications, 2009.

[14] E. Everett, A. Sahai, and A. Sabharwal, "Passive self-interference suppression for Full-Duplex infrastructure nodes," IEEE Transactions on Wireless Communications, vol. 13, no. 2, pp. 680-694, 2014. 